\title{
El trabajo gerencial - del punto de vista de la actividad - en la ejecución de proyectos de ingeniería de gran complejidad: experiencias y tejidos
}

O trabalho gerencial - do ponto de vista da atividade - na implementação de projetos de engenharia de alta complexidade: experiências e tecelagens

Le travail managérial - du point de vue de l'activité - l'exécution des projets d'ingénierie très complexes: expériences et tissages

Managerial work - the point of view of activity - in executing of highly complex engineering projects: experiences and weavings

Rose Mery dos Santos Costa Leite

\section{OpenEdition}

Journals

\section{Edición electrónica}

URL: http://journals.openedition.org/laboreal/2682

DOI: $10.4000 /$ laboreal.2682

ISSN: 1646-5237

Editor

Universidade do Porto

\section{Referencia electrónica}

Rose Mery dos Santos Costa Leite, «El trabajo gerencial - del punto de vista de la actividad - en la ejecución de proyectos de ingeniería de gran complejidad: experiencias y tejidos ", Laboreal [En línea], Volume $12 \mathrm{~N}^{0} 2$ | 2016, Publicado el 01 diciembre 2016, consultado el 24 septiembre 2020. URL http://journals.openedition.org/laboreal/2682 ; DOI : https://doi.org/10.4000/laboreal.2682

Este documento fue generado automáticamente el 24 septiembre 2020.

\section{(c) (1) (5)}

Laboreal está licenciado com uma Licença Creative Commons - Atribuição-NãoComercial 4.0 Internacional. 


\section{El trabajo gerencial - del punto de vista de la actividad - en la ejecución de proyectos de ingeniería de gran complejidad: experiencias y tejidos}

O trabalho gerencial - do ponto de vista da atividade - na implementação de projetos de engenharia de alta complexidade: experiências e tecelagens

Le travail managérial - du point de vue de l'activité - l'exécution des projets

d'ingénierie très complexes: expériences et tissages

Managerial work - the point of view of activity - in executing of highly complex

engineering projects: experiences and weavings

Rose Mery dos Santos Costa Leite

\section{REFERENCIA}

Leite, R.M.S.C. (2014). A atividade gerencial em empreendimentos: experiências e tecelagens. Tese de Doutoramento em Serviço Social, Escola de Serviço Social, Universidade Federal do Rio de Janeiro, Rio de Janeiro.

\section{NOTA DEL EDITOR}

http://dx.doi.org/10.15667/laborealxii0216rcl

Manuscrito recebido em: fevereiro/2016

Aceite após peritagem: setembro/2016 


\section{Introducción}

1 Esta tesis tuvo como campo empírico una empresa pública de Brasil, del segmento de energía, específicamente el sector de petróleo y gas, marcado por una "tradicional" doble cara, pública/privada, que se fue constituyendo, históricamente, por la proximidad del vínculo entre la vida política nacional y la realidad empresarial. Se caracteriza, a veces, como una típica organización de Estado, reforzando su cara pública de ser dinamizadora del desarrollo del país, sin embargo, en otros períodos, lo que se evidencia es su cara privada que está atada a la lógica de la racionalidad empresarial, orientada a la reproducción ampliada de capital. Es una corporación compleja, segmentada, jerarquizada, reconocida internacionalmente por la competencia técnica y un saber-hacer específico (no obstante graves problemas que ha experimentado en los últimos años), que lleva en su cultura rasgos de relaciones basadas en una estructura vertical de poder y orientada por un sesgo disciplinario (Scaletsky, 2003; Leite, 2009; Felipe, 2010).

2 El foco central de la investigación fue tratar de comprender - desde el punto de vista de la actividad - la dimensión de la gestión cotidiana de los gerentes responsables de la implementación de la cartera de proyectos de ingeniería de esta empresa. Tales proyectos tratan de emprendimientos industriales de gran importancia y complejidad, tanto por la dimensión económica y el volumen de negocios, como por la movilización de un enorme contingente de profesionales de las más diversas especialidades, así como una múltiple red de diferentes actores con los más distintos intereses.

Dependiendo de las características de cada proyecto, de su tamaño, grado de complejidad, nivel tecnológico, coeficientes de innovación, indicadores y metas, entre otros requisitos, incluyendo su ciclo de vida (desarrollo, construcción/ejecución y producción/operación), se creará una nueva gerencia en el organigrama con la asignación de realizar la gestión de uno o más proyectos. Se trata de estructuras administrativas transitorias, que están atadas a la temporalidad de los proyectos, variando según la evolución de los mismos, cronogramas previstos, implicaciones legales, licencias, etc. Pueden estar ubicadas en áreas administrativas u operativas, en instalaciones de la propia empresa o de empresas prestadoras de servicios, en el país o en el extranjero, con equipos mixtos formados por trabajadores propios (contratados mediante concurso público), y por prestadores de servicios de terceras empresas. Este escenario implica una dinámica y ritmos propios, un proceso cíclico, porque los equipos están estructurados para la ejecución de un proyecto $\mathrm{y}$, al final de la etapa de conclusión, estas se deshacen completamente.

4 La gestión cotidiana de los proyectos de ingeniería, desde su génesis hasta la etapa de operación de la instalación industrial, está impregnada de numerosas variabilidades e incertidumbres, apoyada por un patrimonio de saberes (científicos y de la práctica) que se fue construyendo a lo largo de la historia de la empresa para compatibilizar exigencias empresariales de ciclos de implantación cada vez más rápidos y de menor demanda de recursos. 


\section{La caja de herramientas teórico-metodológicas}

5 El análisis del trabajo del gerente en empresas que operan sistemas socio-técnicos complejos involucra un campo investigativo y de intervención técnica que asocian, estrechamente, investigación, formación y acción (Teiger, Laville y Duraffourg, 2004). A veces, los saberes desarrollados por los gerentes son "invisibles" para ellos mismos, y se trata, por tanto, de conocimientos "inconscientes e incorporados" y con grandes dificultades de verbalización por los mismos. Se considera que es fundamental tener como presupuesto que "el adulto en formación no es una 'tabla rasa', sino un sujeto 'aprendiz' activo y reflexivo" (Lacomblez y Teiger, 2007, p. 598).

6 La opción teórico-metodológica adoptada tiene como referencia presupuestos éticoepistemológicos de un linaje de pensadores materialistas que comprenden la actividad humana como central en la producción, (re)producción y transformación de las sociedades y los humanos, así como en su desarrollo y en su lucha por la salud.

7 Así, toda situación de trabajo es "siempre en parte, y este en parte es siempre imprevisible, una aplicación de un protocolo y experiencia o encuentro de encuentros" (Schwartz, 2010, p. 42). En este marco, la producción de la tesis recibió las influencias de la perspectiva ergológica (Schwartz, 2000; 2010), y los enfoques clínicos de la Ergonomía de la Actividad (Guérin, Laville, Daniellou, Duraffourg, y Kerguelen, 2001; Falzon, 2007), la Psicodinámica del Trabajo (Dejours, 2012; 2013), y la Clínica de la Actividad (Clot, 2006; 2010) que permitió llevar al campo de debates la articulación macro-micro en el análisis de las actividades presentes en el trabajo del gerente, cuyas normas están poderosamente atravesadas por un mundo de intereses y valores conflictivos.

8 La proposición es que la caja de herramientas tenga referencias para pensar otro modo de abordar el trabajo de los gerentes. Parte de la premisa de que toda construcción teórico-metodológica, al mismo tiempo que exige rigor científico, no se da a priori, ni sin tener en cuenta las condiciones singulares del estudio en cuestión y el campo de relaciones en que se da. El análisis no tiene la pretensión de agotarlas, sino de promover la interlocución de los conceptos que pueden contribuir a la comprensión del trabajo gerencial, desde el punto de vista de la actividad.

9 Es de destacar la vinculación institucional de la autora de la tesis al campo empírico, dado que era gerente sectorial en la empresa en cuestión. Esta implicación (Lourau, 1990), considerando la condición híbrida de investigadora y gerente, ha resultado ser un importante ingrediente en el proceso, por una parte requiriendo una permanente atención al rigor epistemológico y teórico-metodológico, por otra resultando un factor determinante para la producción de conocimiento.

10 Durante el itinerario de investigación, considerando las particularidades del terreno y sus protagonistas, el posicionamiento de hibridismo de la investigadora y los objetivos de la tesis, la identificación de un enfoque metodológico que congregara estos factores ha sido un tema que ha contribuido al debate sobre la adopción de una alternativa metodológica, junto a los gerentes, que no se limitara a la recogida de datos, sino que suscitara el establecimiento de una relación dialógica movilizadora de la expresión de las experiencias vividas de su propia actividad.

11 El Método de la Reconstitución Biográfica Centrada en el Trabajo, concebido por Ramos (2010), fue la opción vislumbrada en aquel momento porque se mostraba impulsor de la 
dinámica deseada entre la investigadora y los gerentes. Fue potenciador para la comprensión de las situaciones de trabajo, en la medida en que se configuró como un proceso de intervención, en un enfoque clínico (dado que partió de cuestiones que se enmarcan en el trabajo específico, pero que lo superaban), suscitando el surgimiento de significados y sentidos de las vivencias cotidianas. El método se basa en la articulación entre dos instrumentos que se reconfiguraron a la luz de las especificidades de la tesis, tales como: a) las "Entrevistas de Reconstitución Biográfica" (Ramos, 2010) que corresponden a una forma de entrevista semiestructurada, de carácter biográfico, y se realizan, con el mismo gerente, diferentes entrevistas. En el sentido de registrar una diferenciación con respecto a las técnicas que hegemónicamente se emplean en este terreno, se adoptó la denominación de "conversación" [1], en lugar de "entrevista". En la primera conversación se estableció un acuerdo de trabajo/de cooperación, se ratificó la propuesta de estudio, se establecieron las bases de confidencialidad, se presentó el dispositivo de investigación y se inició la conversación. En las conversaciones siguientes, que tuvieron una variabilidad de una a tres, a partir de la restitución de la conversación anterior, los diálogos prosiguieron, recorriendo la línea de tiempo, pero no necesariamente de manera lineal. La "Entrevista de Reconstitución Biográfica" tiene la finalidad de (re)construir el curso de vida, donde se eligen las experiencias vividas y "archivadas" relacionadas con la realidad actual vivida (Ramos, 2010), dado que la "biografía está siempre completa sin el jamás estar" (Ramos, 2010); b) un dispositivo, concebido como un soporte gráfico, con la función de ser una guía orientadora y de apoyo a la realización de las conversaciones que recibió en esta investigación la denominación de "Trama de la Vida". Este dispositivo incluye dos tipos de datos: los "heterobiográficos y autobiográficos" (Ramos, 2010, p. 110). Los datos "heterobiográficos" se toman de fuentes documentales como, por ejemplo, informes, sistemas y otros mecanismos que estén relacionados con el tema tratado. Y los datos "autobiográficos", que se derivan de las conversaciones junto a los protagonistas dedicados a la investigación.

La estructura central del dispositivo "Trama de la Vida" parte de una línea de tiempo, donde se agrupan bloques de líneas horizontales tanto en la parte superior como inferior del tiempo delimitado. Se abordaron nueve temas en este dispositivo para atender a los objetivos del estudio, siendo tres de ellos referidos a los datos "heterobiográficos" (recorrido de actualización formativa, recorrido preparatorio y recorrido gerencial) y seis relativos a los datos "autobiográficos" (actividades diarias/ ejes operativos, proyectos, dificultades/facilidades, equipos, historia de salud y referencias). El dispositivo "Trama de la Vida" se constituyó como un facilitador para la reconstitución biográfica por presentar una estructura y lenguaje visual que está referido a una lógica más tecnicista y a rasgos identitarios, habituales en los procesos de trabajo de estos gerentes.

La proposición de investigación se sometió a la aprobación de la empresa que destacó la relevancia de la temática y la originalidad del dispositivo.

El universo abarcado por la investigación empírica totalizó 53 gerentes con la asignación de implementación de empresas, todos concursados con, al menos, licenciatura en ingeniería, en las más diversas especialidades, siendo el $81 \%$ con edad entre 40 y 62, y el 71\% con más de 19 años de permanencia en la empresa. Participaron en la investigación siete gerentes, del sexo masculino, con gran experiencia, variando de 27 a 38 años de trabajo en la empresa y de 14 a 25 años en el ejercicio gerencial. Para 
resguardar la confidencialidad de estos gerentes no fue posible especificar demás información sobre el perfil. Se registraron todas las conversaciones, en audio, con previa autorización de los mismos.

Las invitaciones se hicieron presencialmente, por contacto telefónico o por correo, y en estos momentos, se presentaron la justificación y los objetivos del estudio.

Se construyeron siete diferentes dispositivos "Trama de la Vida", dado que estos son instrumentos individuales.

17 El "Método de la Reconstitución Biográfica Centrada en el Trabajo" se mostró una vía potenciadora de movilización subjetiva, en la medida en que los diálogos que se produjeron no se refieren a relatos de experiencias a partir de una trayectoria de trabajo lineal, creando posibilidades de que se puedan (re)contar las respectivas historias narradas basándose en nuevas elaboraciones, explicaciones y relaciones con otros hechos o eventos ocurridos, que se reverberan con la realidad vivida y actual.

Todo material procedente de las conversaciones fue transcrito íntegramente, con el fin de garantizar el máximo de fiabilidad a los registros constantes en los audios.

\section{Análisis y discusión de los resultados}

19 En el proceso analítico se buscó poner en sinergia las referencias presentadas en el marco teórico-metodológico y el análisis de los materiales presentes en los discursos de los gerentes, centrándose en el contexto dialógico. Implicó la constitución de varias categorías emergentes de los diálogos, en las que se activaron los nueve campos temáticos del dispositivo "Trama de la Vida".

20 El análisis muestra referencias sobre los diversos modos de reconstrucción individual y colectiva de estos gerentes en el transcurso de la historia de la empresa y la gestión de los proyectos de ingeniería; destacó la importancia de la naturaleza nómada de la actividad determinada por los proyectos de ingeniería; indicó el distanciamiento entre las prescripciones/la estructura técnica y las actividades; evidenció la multiplicidad de los engendramientos que involucran la gestión de contratos y los equipos; presentó la diversidad de recursos lenguajeros de naturaleza epopéyica como una alternativa para expresar la gestión de la actividad gerencial cotidiana; y propició que se especificaran las fuentes de inspiración presentes y actuales.

21 Los resultados muestran que se trata de un trabajo cuyas actividades requieren disponibilidad de los profesionales para estar, a lo largo de los años de profesión, en un permanente proceso territorial migratorio. Esta característica impacta en las relaciones sociofamiliares, demandando que toda la vida esté regida por esta desterritorialización sistemática. Por parte de los gerentes, hay una búsqueda de naturalización de este proceso, que señala la adopción de una estrategia defensiva que intensifica el valor y la importancia del trabajo realizado y las respectivas ganancias derivadas, minimizando, por consiguiente, los costes originados de la complejidad de las situaciones y los impactos negativos en sus vidas.

22 Cada gerente ha encontrado modos singulares de aprender con las exigentes experiencias migratorias a las que se ha enfrentado. En la gestión de sus actividades trató de compatibilizar la atención a los requisitos de calidad y las expectativas empresariales $\mathrm{y}$, al mismo tiempo, hacer frente a los fracasos ante los desafíos 
plateados y la dinámica de la vida fuera del trabajo, en sus dimensiones simbólicas, relacionales y psicológicas.

Los diálogos resaltan sobre la presencia de un cuestionamiento sobresaliente en relación con la política empresarial, en curso, que está amplificando y valorando la lógica procedimental más allá de lo que se requiere técnicamente. Indican que este es un proceso que genera descalificación y vaciado de la complejidad imbricada en las actividades gerenciales destacadas, tratándolas de manera simplista, como si fuera posible acceder, mediante un manual, a toda la movilización de la inteligencia astuta existente en la actividad humana. Se trata de negar la riqueza de la potencia de la vida o de igualarla a un modo o a una rutina, en los que no es necesario que exista el trabajo vivo.

Los gerentes, cotidianamente, se enfrentan a situaciones de trabajo variadas, llenas de imprevisibilidades, a partir de las cuales deben comprometerse, detectando/ interpretando/evaluando las cuestiones emergentes, creando soluciones, inventando artimañas e incorporando sutilezas. La inteligencia movilizada frente a las situaciones reales de trabajo y que engendra soluciones inéditas para los problemas, las variabilidades y los imprevisibles exigen celo (Dejours, 2012; 2013). Esta inteligencia creadora, enraizada en todo el cuerpo en la relación sensorial y perceptual del cuerpo (no sólo del cerebro) con la tarea se refiere a la malicia, la perspicacia, la astucia, que en la mitología griega se refiere a la diosa Metis [2] (Dejours, 2012; Detienne, y Vernant, 2008). Algo que Schwartz $(2000 ; 2011)$ ha denominado cuerpo-sí.

En este sentido, la gestión de contratos, pese a estar regida por prescripciones determinantes en esta actividad, demanda, desde el punto de vista de sus protagonistas, un tipo de inteligibilidad concebida en la vivencia de la práctica y un proceso de formación que se va configurando y aprendiendo, paulatinamente, a lo largo de la experiencia adquirida en cada proyecto desarrollado. Un patrimonio fruto de este campo experiencial que incluye varios aspectos, tales como: a) multiplicidad y complejidad de contratos que involucran a varias terceras empresas nacionales y/o internacionales, prestadoras de servicios hiperespecializados; b) cuestiones técnicas relacionadas con indicadores cuantificables, de conformidad con las métricas nacionales y/o internacionales, específicas del negocio; y c) decisiones de los niveles jerárquicos superiores, que implican la integración de múltiples temáticas, de ámbito interno y externo, considerando, entre otras, la naturaleza pública de la empresa contratante, terreno de la investigación. Se trata, cotidianamente, de relaciones con empresas contratadas que indican permanentes y contundentes debates de normas, en los que circula un mundo de valores. Fue posible comprender que la experiencia de estos gerentes subraya la exigencia de una actividad dialógica que se debe priorizar para el establecimiento de negociaciones cotidianas imprescindibles, de modo que se puedan superar los problemas sistemáticamente, tomando en cuenta la singularidad de cada situación de trabajo y los métodos operatorios y de gestión que serán (re)normatizados (Schwartz, 2000) y (re)normalizados.

Pese al modelo gerencial vigente, atravesado por expresiones de autoritarismo, dar señales de hegemonía, los gerentes marcan en sus discursos posiciones divergentes entre sus respectivas visiones, que priorizan el diálogo y el debate y las prácticas en curso. Las narrativas presentan un trabajo atravesado por varios atributos de pionerismo, fuerza, capacidad de "hacer la guerra y de sentirse Dios", símbolos que hacen referencia a la virilidad (y a sistemas defensivos psíquicos por ella marcados), al 
mismo tiempo que requieren astucia, una inteligencia virtuosa y polarizada por lo femenino (Dejours, 2012). Los diálogos subrayan, además de las normas antecedentes, las alternativas fijadas procedentes de la inteligencia astuta, destacándose la dimensión colectiva del trabajo, representada por las relaciones de cooperación. Así configurando caminos estratégicos posibles para aumentar la fiabilidad junto a los demás gerentes, los equipos y las empresas prestadoras de servicios, de modo que la inversión en este tejido pudiera contribuir al objetivo mayor de estos gerentes: "tener la obra terminada dentro de lo previsto".

\section{Consideraciones finales}

Ante situaciones reales de trabajo, estos gerentes revelan actualizar su actuar en competencia (Schwartz, 2010), desarrollando y movilizando otras, encontrando salidas y soluciones para los imprevistos y las variabilidades. Los diálogos están delimitados por cuestiones vinculadas a una movilización subjetiva producida y por un lenguaje gerencial metafórico, en los que definen la ejecución de los proyectos de ingeniería como un verdadero "campo de batalla o de guerra" y se autodenominan de distintas formas: como "Dios", “César, el emperador", o el personaje "héroe de guerra, Rambo".

Los materiales discursivos de estos gerentes tratan de las experiencias vividas a lo largo de la implementación de diferentes proyectos de ingeniería que engendran una trayectoria gerencial no lineal, sino adentrada en una verdadera "trama". Este tejido fue estructurado por rasgos de una gestión autoritaria y jerarquizada, que permeó el proceso histórico de la empresa y las interferencias socioeconómicas, políticas y culturales del país. Se trata de un cotidiano vivo marcado por características peculiares y adversas de la naturaleza del negocio, relativo a la industria extractiva, "sacar riquezas energéticas, perforando y adentrando en las entrañas de la tierra". Y también por diálogos de enfrentamientos que asumen el diseño de un escenario aguerrido y bélico, establecidos por expresiones y metáforas que indican la faceta gerencial como un "combatiente", relacionado con un tipo de resignación heroica y una actividad lenguajera, fruto de una producción social e histórica vivida.

Los protagonistas de las actividades en cuestión destacan la riqueza y fecundidad del proceso de aprendizaje y desarrollo a lo largo de sus trayectorias profesionales, dado que estas no se limitan a lo que se lleva a cabo, sino también a las dificultades y enfrentamientos, los riesgos, a un desarrollo histórico que teje una trama que se entrelaza entre las dimensiones profesional y personal y que privilegia las respectivas vivencias. Las relaciones dialógicas están siempre presentes, y de forma permanente, con otros gerentes que ya tenían alguna experiencia para construir un proceso evolutivo durante la implantación de una obra. Destacan que en un proceso de formación futura que se llevará a cabo, el lenguaje y la comunicación deben ocupar un lugar destacado en la producción de saberes, considerando la manera en que se dicen las cosas para buscar comprenderlas cómo son pensadas y concretadas (Santos, y Lacomblez, 2001/2002).

El enfoque centrado en el itinerario de vida y de trabajo en este estudio se configuró como un proceso de intervención, como un método clínico, en la medida en que partió de cuestiones que se enmarcan en el trabajo. Este recurso teórico-metodológico puede constituirse en una vía de acceso a otras investigaciones en este terreno o en otros de la 
misma naturaleza, que también estén atravesados por una lógica racional y cuantitativa.

El dispositivo "Trama de la Vida", por tener una "arquitectura" que lo vinculó a la realidad de trabajo de estos gerentes, ha creado condiciones para que los diálogos circularan sin extrañeza y como un espacio disparador de una movilización subjetiva. La narrativa del "contar" les llenó de orgullo por el trabajo realizado y por las estrategias activadas durante sus enfrentamientos, dado que nadie se había interesado y les había pedido para "contar" sus historias hasta aquel momento.

Explorar el trayecto de vida en el trabajo, retomando vivencias pasadas, representó para estos gerentes una primera experiencia de confrontación consigo mismo y con los eventos que se destacaron a lo largo de las conversaciones, caracterizándose como un estímulo a la reflexión, el intercambio y el diálogo sobre la experiencia vivida y vívida traída a la memoria y la realidad actual, lo que pone en evidencia la potencialidad de este estudio.

Sus resultados sugieren que se revise el proceso de formación gerencial, teniendo en cuenta los referenciales de la literatura de la Administración y las "ciencias de la gestión" de base conceptual mecanicista y tecnocrática, así como la adopción de referencias cognitivo-conductuales para abordar las cuestiones relativas a los humanos. Los diálogos expresan que la vía de formación gerencial empleada por la empresa se muestra insuficiente para cumplir las metas de productividad, seguridad y calidad en el trabajo, ante las exigencias y complejidades de las situaciones reales. Los gerentes señalan la necesidad de constitución de caminos que promuevan el debate, que reúnan los esfuerzos colectivos y que pongan en sinergia el "trabajar juntos" en situaciones de trabajo.

\section{BIBLIOGRAFÍA}

Clot, Y. (2006). A Função Psicológica do Trabalho. Petrópolis-RJ: Vozes.

Clot, Y. (2010). Trabalho e Poder de Agir. Belo Horizonte: Fabrefactum.

Dejours, C. (2012). Trabalho vivo. Brasília: Paralelo 15.

Dejours, C. (2013). o fator humano. Rio de Janeiro: FGV, $2^{a}$ ed.

Détienne, M., \& Vernant, J. (2008). Métis: as astúcias da inteligência. São Paulo: Odysseus.

Falzon, P. (2007). Ergonomia. São Paulo: Edgard Blücher.

Felipe, E. S. (2010). Mudanças institucionais e estratégias empresariais: a trajetória e o crescimento da Petrobras a partir da sua atuação no novo ambiente competitivo (1997-2010). Tese de Doutorado.

Economia. Universidade Federal do Rio de Janeiro, Rio de Janeiro.

Guérin, F., Laville, A., Duraffourg, J., \& Kergelen, A. (2001). Compreender o trabalho para transformálo: a prática da Ergonomia. São Paulo: Edgard Blücher. 
Lacomblez, M., \& Teiger, C. (2007). Ergonomia, formações e transformações. In: P. Falzon (Org.). Ergonomia (587-599). São Paulo: Edgard Blücher.

Leite, R. M. S. C. (2009). Bandeirantes do Mar: a identidade dos trabalhadores das plataformas de petróleo. Rio de Janeiro: Intertexto.

Lourau, R. (1990). Implication et surimplication. Revue du Mauss, n.10.

Ramos, S. (2010). Envelhecimento, Trabalho e Cognição: do laboratório para o terreno naconstrução de uma alternativa metodológica. Lisboa: Fundação Calouste Gulbenkian.

Santos, M., \& Lacomblez, M. (2001/2002). Discutir o trabalho, fazer sabendo: Projecto de formação profissional de adultos. Cadernos de Consulta Psicológica, 17/18, 305-311.

Scaletsky, E. C. (2003). o patrão e o petroleiro : um passeio pela história do trabalho na Petrobras. Rio de Janeiro: Relume Dumará.

Schwartz, Y. (2000). Le paradigme ergologique ou un métir de Philosophe. Toulouse: Octarès.

Schwartz, Y. (2010). Trabalho e Ergologia. In Y. Schwartz, \& L. Durrive (Orgs.) Trabalho e Ergologia: conversas sobre a atividade humana. Niterói-RJ: EDUFF, $2^{\mathrm{a}}$ ed. ampliada.

Schwartz, Y. (2011). Pourquoi le concept de corps-soi? Corps-soi, activité, expérience. Travail et Apprentissages, 7, 148- 177.

Teiger, C., Laville, A. , \& Duraffourg, J. (2004). Trinta anos depois: reflexão sobre uma história das relações entre pesquisa em ergonomia e ação sindical na França (o caso das operárias do setor eletrônico entre 1965 e 1975). In M. Figueiredo, M. Athayde, J. Brito, \& D. Alvarez. (Orgs.)

Labirintos do trabalho: interrogações e olhares sobre o trabalho vivo. Rio de Janeiro: DP\&A.

\section{NOTAS}

1. Athayde (2010, en comunicação pessoal) prefiere denominar "conversaciones sobre el trabajo" a lo que también se llama "entrevistas dialógicas". Mientras la entrevista está atravesada por cierto "grado" de formalidad, la conversación presupone algo más informal, intuitivo, fuertemente relacional, haciendo referencia a un encuentro o una "charla".

2. Según la Psicología Histórica, los estudios de Detienne y Vernant (2008) sobre la Antigua Grecia muestran en la mitología la figura de la diosa Metis, caracterizada por los fecundos consejos prácticos, dada su astucia, a Zeus (de quien habría sido su primera esposa). Este concepto se vincula a la figura de lo femenino y se refiere a un tipo de inteligencia que aborda la interpretación, la improvisación, la astucia, la creación, la invención, el disfraz, la subversión y la transgresión. Es una inteligencia de la acción que demanda el compromiso de todo el cuerpo y subjetividad, en el esfuerzo de enfrentamiento de las situaciones ante lo inesperado. 


\section{AUTOR}

\section{ROSE MERY DOS SANTOS COSTA LEITE}

Petróleo Brasileiro S.A. - Avenida Henrique Valadares, 28, 9o andar, Centro, Rio de Janeiro, Brasil, CEP 20.231-030

rose.milk@yahoo.com.br 